ARTICLE

https://doi.org/10.1038/s41467-021-26451-0

\title{
Writing of strain-controlled multiferroic ribbons into $\mathrm{MnWO}_{4}$
}

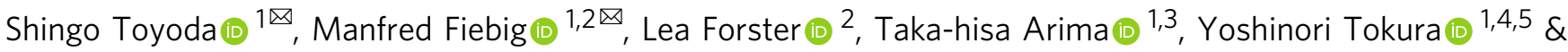 \\ Naoki Ogawa, $1,5,6$
}

Local and low-dimensional structures, such as interfaces, domain walls and structural defects, may exhibit physical properties different from the bulk. Therein, a wide variety of local phases were discovered including conductive interfaces, sheet superconductivity, and magnetoelectric domain walls. The confinement of combined magnetic and electric orders to spatially selected regions may be particularly relevant for future technological applications because it may serve as basis of electrically controllable magnetic memory devices. However, direct observation of magnetoelectric low-dimensional structures cannot readily be done partly because of the lack of experimental techniques locally probing their physical nature. Here, we report an observation of multiferroic ribbon-like domains in a non-multiferroic environment in $\mathrm{MnWO}_{4}$. Using optical second harmonic generation imaging, we reveal that a multiferroic phase is stabilized by locally generated strain while the bulk magnetic structure is nonmultiferroic. We further find that the confined multiferroic state retains domains with different directions of electric polarization and we demonstrate deterministic writing of a multiferroic state embedded in a non-multiferroic environment.

\footnotetext{
${ }^{1}$ RIKEN Center for Emergent Matter Science (CEMS), Saitama 351-0198, Japan. ${ }^{2}$ Department of Materials, ETH Zurich, 8093 Zurich, Switzerland.

${ }^{3}$ Department of Advanced Materials Science, University of Tokyo, Kashiwa 277-8561, Japan. ${ }^{4}$ Tokyo College, University of Tokyo, Tokyo 113-8656, Japan.

${ }^{5}$ Department of Applied Physics, University of Tokyo, Tokyo 113-8656, Japan. ${ }^{6}$ PRESTO, Japan Science and Technology Agency (JST), $332-0012$

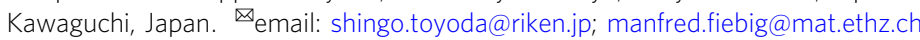


$\mathrm{n}$ geometrically frustrated magnets, magnetic order is suppressed due to competing magnetic interactions, and nontrivial magnetic structures such as spin-liquids ${ }^{1}$, skyrmions ${ }^{2}$, and spin-spirals ${ }^{3}$ may emerge. Among them, incommensurate non-collinear spin structures are of particular interest as they can show spin-induced electric polarization and pronounced magnetoelectric cross-coupling 4,5 . As mentioned above, local structures $^{6-10}$ can exhibit new local phases ${ }^{11,12}$ so that interest has been shifting from bulk multiferroics towards multiferroicity confined to the lower dimensions with multiferroic domain walls as a prime example ${ }^{13,14}$.

We report an observation of deterministic multiferroic ribbons in a non-multiferroic environment in $\mathrm{MnWO}_{4}$ using optical second harmonic generation (SHG) as an imaging technique. Even though the bulk magnetic structure features a collinear non-multiferroic spin order, a multiferroic spin-spiral phase is locally stabilized due to the strain caused by surface defects in the crystal lattice. Within the multiferroic ribbons, we find polarization domain states in line with the ferroic nature of the spatially restricted phase. We propose a model explaining such a confined multiferroic state in a non-multiferroic environment, and we demonstrate multiferroic writing of information.

\section{Results}

$\mathrm{MnWO}_{4}$ crystallizes in the monoclinic wolframite structure with the centrosymmetric space-group $P 2 / c$ (point group: $2 / \mathrm{m}$ ). The lattice constants $a, b, c$, and $\beta$ are $4.82,5.75,4.99 \AA$ and $91^{\circ}$, respectively ${ }^{15}$. In the following, we use Cartesian coordinates $x \|$ $a, y\|b, z\| c$, to represent the crystallographic axes, since $\beta \approx 90^{\circ}$. The magnetically active ion is $\operatorname{Mn}^{2+}(S=5 / 2)$, which forms zigzag chains along the $z$ axis, as shown in Fig. 1a. Several higher-order magnetic interactions are known to compete with one another, resulting in magnetic frustration ${ }^{16}$. Successive magnetic phase transitions below the Néel temperature $\left(T_{N}=13.5 \mathrm{~K}\right)$ reflect the presence of several near-degenerate antiferromagnetic phases. Coming from lower temperatures, these antiferromagnetic phases are called AF1 (collinear, commensurate, Fig. 1b), AF2 (elliptical spiral, incommensurate), and AF3 (collinear, incommensurate). Although the magnetic structures in the AF1 and AF3 phases retain the centrosymmetric point group $2 / \mathrm{m}$ symmetry, the spin-spiral structure in the AF2 phase lowers the magnetic point group to noncentrosymmetric 2 . A magnetically induced ferroelectric polarization which is explained by the spin current model ${ }^{17}$ or an inverse Dzyaloshinskii-Moriya effect ${ }^{18}$ appears along the $y$ axis in the multiferroic AF2 phase (Fig. 1c) ${ }^{19,20}$, whereas the AF1 and AF3 phases are not multiferroic.
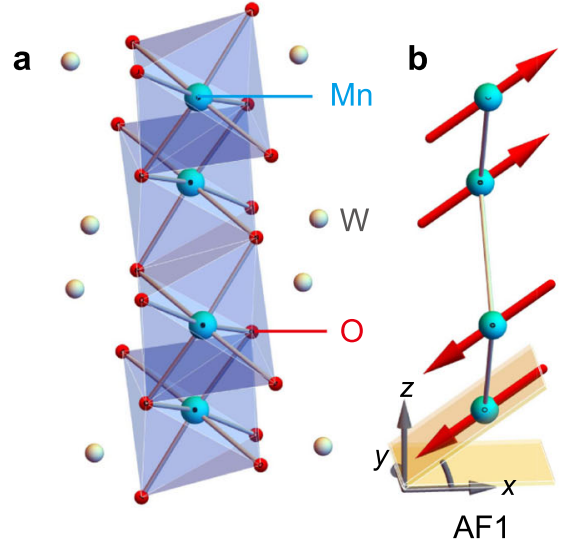

d

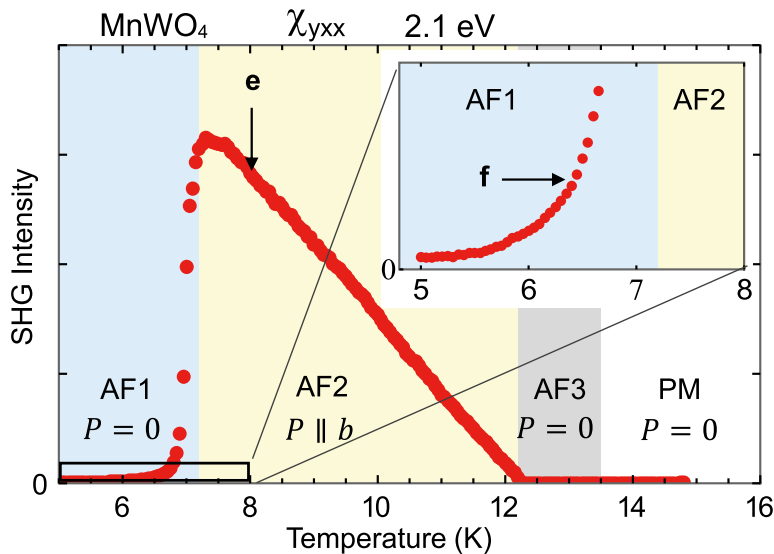

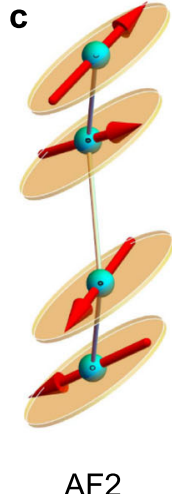

AF2

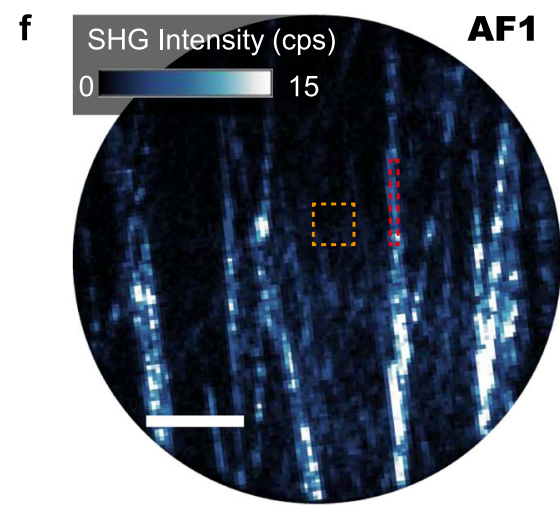

Fig. 1 Temperature dependence of second harmonic generation. a Crystal Structure of $\mathrm{MnWO}_{4}$. b, c Magnetic structures in the $\mathbf{b}$ AF1 and $\mathbf{c}$ AF2 phases, respectively. The red arrows represent the magnetic moments of the $\mathrm{Mn}^{2+}$ ions and yellow circles show the spin-spiral plane. $\mathbf{d}$ Temperature dependence of the SHG for the tensor component $\chi_{y x x}$ at the photon energy $2 \hbar \omega=2.1 \mathrm{eV}$. The inset shows the enlarged view of the SHG intensity in the SHG-forbidden AF1 phase. e The SHG image was obtained at $8 \mathrm{~K}$ in the multiferroic AF2 phase after the zero-field cooling. $\mathbf{f}$ The SHG image was obtained at $6.5 \mathrm{~K}$ in the non-multiferroic AF1 phase. The orange and red regions indicate the regions which are used for the polarization dependence measurements in Fig. $2 \mathrm{~d}$. Scale bars, $200 \mu \mathrm{m}$. 
Our experiment relies on spatially resolved imaging by SHG, that is, frequency-doubling of a light wave in a material. This is expressed by $\mathbf{S}_{\mathrm{i}}(2 \omega)=\epsilon_{0} \chi_{i j k} \mathbf{E}_{\mathrm{j}}(\omega) \mathbf{E}_{\mathrm{k}}(\omega)$, where $\mathbf{S}(2 \omega), \chi_{i j k}$, and $\mathbf{E}(\omega)$ are the source of the frequency-doubled light, the SHG susceptibility, and the electric field of the incident light, respectively. In the leading electric-dipole order, $\chi_{i j k}$ becomes nonzero in noncentrosymmetric materials only, which makes SHG a particularly powerful technique to detect the multiferroic state because of the associated breaking of spatial inversion symmetry. Therefore, only the AF2 phase permits an ED-SHG signal for which the tensor components $\chi_{y x x}, \chi_{y y y}$, and $\chi_{x y x}$ are nonzero with light propagating along the $\mathrm{z}$ axis ${ }^{21,22}$. Figure $1 \mathrm{~d}$ shows the temperature dependence of the SHG signal of a (001)-oriented polished $\mathrm{MnWO}_{4}$ platelet at the photon energy $2 \hbar \omega=2.1 \mathrm{eV}$ for $\chi_{y x x}$, which couples to the ferroelectric polarization along the $y$ axis ${ }^{23}$. A large SHG signal whose amplitude scales with the spontaneous polarization is obtained in the multiferroic AF2 phase. An SHG signal, in total on the order of $1 \%$, remains in the non-polar AF1 phase, see Fig. 1d. This residual signal cannot be associated with trivial sources such as surface SHG, luminescence, or higher-order non-polar SHG contributions, because it is absent in the paramagnetic and the AF3 phases. Therefore, the "forbidden" SHG signal likely is of magnetic origin and suggests the existence of a polar magnetic sub-structure in the nonmultiferroic AF1 phase.

We perform SHG imaging measurements to understand the origin of the forbidden signal. Figure $1 \mathrm{e}, \mathrm{f}$ show spatially resolved SHG images at $2.1 \mathrm{eV}$ in the multiferroic AF2 phase and in the non-multiferroic AF1 phase, respectively. In the AF2 phase (Fig. 1e), a ferroelectric multidomain structure with bulk domains of a few hundred micrometers extension in all three dimensions is formed $^{24}$. The different levels of brightness result from the interference of the SHG light waves from vertically arranged $+P$ and $-P$ domains. The SHG image in the AF1 phase (Fig. 1f) reveals that the forbidden SHG signal comes from line-shaped structures ("ribbons"). As mentioned, their polar nature must be related to magnetic order, because the associated SHG signal disappears in the paramagnetic phase. We thus reveal the existence of spatially confined polar magnetic regions in a non-polar magnetic bulk environment.

To further elucidate the origin of the ribbons, we performed SHG spectroscopy and polarization anisotropy measurements. Figure 2 a shows the SHG spectra for the tensor component $\chi_{y x x}$ at three different temperatures along with a linear optical absorption spectrum in the paramagnetic phase. The large SHG peak at $1.97 \mathrm{eV}$ in the multiferroic AF2 phase is caused by optical phase matching 25 , whereas the structure at higher photon energy is assigned to intra-atomic spin-forbidden $d$ - $d$ electronic transitions of the $\mathrm{Mn}^{2+}$ ion ${ }^{26}$. Although the former signal stems from the entire thickness of the sample, the latter one is emitted only from a region near the surface because of the finite coherence length and the optical absorption band above $2 \mathrm{eV}$. Strikingly, the phasematched SHG peak at $1.97 \mathrm{eV}$ from the entire sample is reduced by three orders of magnitude in the AF1 phase, suggesting that only $0.1 \%$ of the crystal volume continues to emit AF2-like SHG and stemming from the near-surface region. In contrast, the signal at higher photon energy is reduced by only one order of magnitude. This suggests that the SHG signal in the AF1 phase stems from the near-surface region. This would locate the magnetic polar ribbons in the AF1 phase at this surface to which they appear to be pinned in some form. We show in Fig. 2b, a line
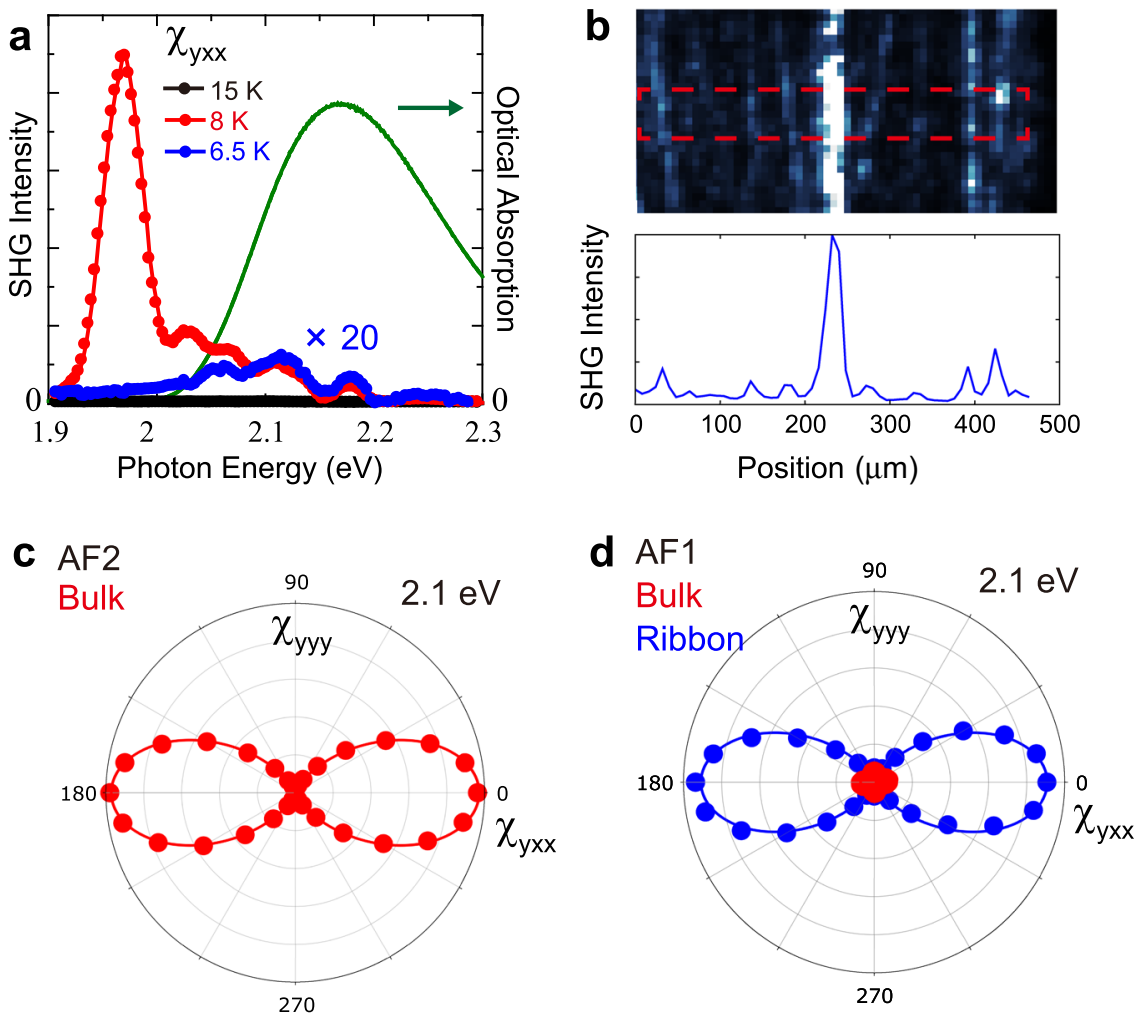

Fig. 2 SHG spectrum, line profile, and polarization dependence. a SHG spectrum measured for the tensor component $\chi_{y x x}$. The green line shows the optical absorption spectrum measured at $15 \mathrm{~K}$. b Line profile (red outline) of the SHG intensity at $6.5 \mathrm{~K}$ in the AF1 phase. c and d SHG polarization anisotropy with the polarization of $E^{2 \omega}|| y$ measured with varying polarization of fundamental light in the $\mathbf{c}$ multiferroic AF2 phase and $\mathbf{d}$ non-multiferroic AF1 phase. The SHG intensity from the AF1 dark bulk region (red) is one order of magnitude weaker than that in the AF1 multiferroic ribbons (blue). Solid lines are fits of the tensor component $\chi_{y x x}$ 
profile of the SHG intensity in the AF1 phase. The SHG intensity in between ribbons is more than an order of magnitude weaker than that in the ribbons in agreement with the bulk-AF1-SHGinactive collinear magnetic structure.

In the anisotropy plots in Fig. $2 \mathrm{c}$ and d, we show the polarization dependence of the SHG signal, which was measured by rotating the polarization of incident light while detecting frequency-doubled component polarized parallel to the $y$ axis. Figure $2 \mathrm{c}$ shows the anisotropy in the AF2 phase, whereas Fig. $2 \mathrm{~d}$ probe the AF1 phase. The SHG anisotropy in the bulk region (red dots) in Fig. $2 \mathrm{~d}$ is obtained on the area of the dark bulk region (orange outline in Fig. 1f), whereas the SHG anisotropy of the ribbons (blue dots) is obtained on the area of the bright line-like region (red outline in Fig. 1f). In the multiferroic AF2 phase, the magnetically induced ferroelectric polarization along the $y$ axis manifests as an SHG signal from the $\chi_{y x x}$ component, see Fig. 2c. Exactly the same type of SHG anisotropy was observed for the polar ribbons in the non-polar AF1 phase, which suggests an AF2-like confined phase with a spontaneous electric polarization along the $y$-axis as its origin. In contrast, the SHG signal in the dark AF1 bulk region is negligibly weak compared with that of the ribbons. This is in perfect agreement with the centrosymmetric magnetic structure of the AF1 phase where the leadingorder electric-dipole SHG is forbidden ${ }^{23}$.

The observed AF2-like nature of the ribbons in the AF1 phase indicates that a sort of phase pinning takes place at the surface. It is possible that the mechanism stabilizing this pinning in the AF1 phase is still active in the AF2 phase at higher temperatures. We, therefore, investigated the relationship between the AF2-like regions and the actual AF2 phase with the experimental setup shown in Fig. 3a. As in Fig. 1, we probe our sample in the quasiphase-matched regime ${ }^{27}$. Figure $3 \mathrm{~b}$ shows a spatially resolved
SHG image obtained after cooling in an electric field $E=+240 \mathrm{kV} / \mathrm{m}$ along the $y$ axis. The homogeneous distribution of the SHG intensity indicates a single-domain state in which the ribbons have seemingly been lost. In a reversal of the electric field subsequent to the field cooling procedure, however, the polar ribbons reappear as bright stripes, see Fig. 3c. The opposite brightness indicates the electric polarization of the ribbon is now opposite from the polarization in the bulk. Hence, we find that the bulk polarization is flipped with the reversal of the electric field while the polarization of the ribbons remains parallel to the initial polarization direction $+P$. It demonstrates that the ribbons are robust against the external electric field, which supports the aforementioned conclusion of surface pinning.

Next, we investigate the electric field dependence of the SHG image after zero-field cooling (ZFC). As depicted in Fig. 3d, a multidomain state is formed in the multiferroic AF2 phase after ZFC, and the ribbons appear to have vanished. When we apply an electric field $E=+240 \mathrm{kV} / \mathrm{m}$, however, the upper part of the ribbons reappears whereas the lower part remains absent. Upon field reversal, the lower part of the ribbons becomes visible whereas the upper part vanishes. These results indicate that, most strikingly, the upper part of the ribbons has the $-P$ polarization, whereas the lower part has the $+P$ polarization after ZFC. Apparently, the ribbons can feature domains with opposite directions of the electric polarization. Since the existence of domains is a hallmark of ferroic order, their observation clearly shows that the polar ribbons in the AF1 phase can in fact be associated with a true ferroelectric and, hence, multiferroic phase. A possible mechanism that could stabilize such a state near the surface is strain. It was reported that the AF1-AF2 phase transition temperature is strongly affected by hydrostatic pressure, suggesting a large spin-lattice coupling in $\mathrm{MnWO}_{4}{ }^{28}$. Hence,
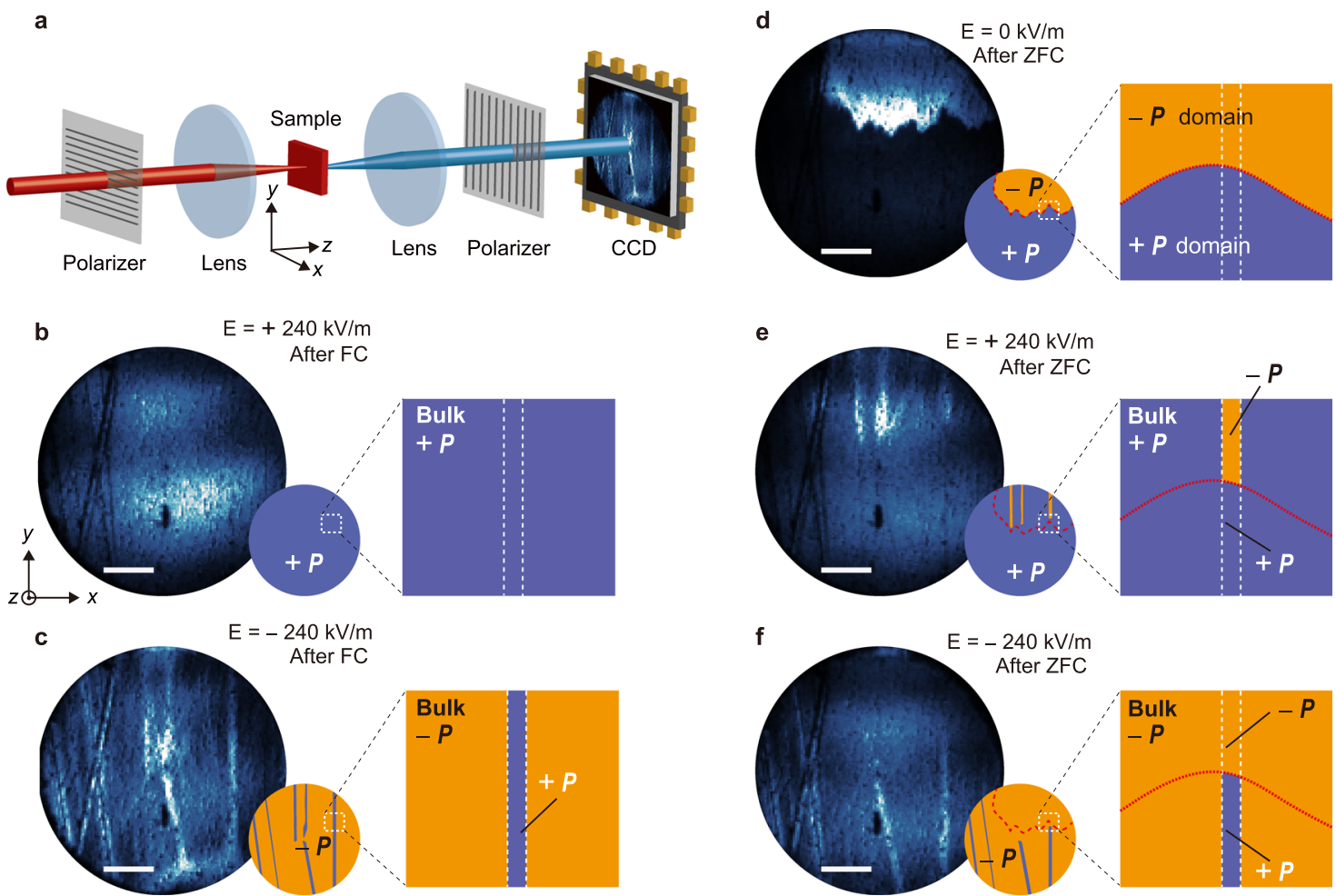

Fig. 3 Multiferroic nature of the ribbons in the bulk multiferroic AF2 phase. a Schematic of the experimental setup for SHG imaging measurements. The incident light with the photon energy $\hbar \omega=1.05 \mathrm{eV}$ was polarized along the $x$ axis. The SHG light with the polarization of $E^{2 \omega} \| y$ (tensor component $\chi_{y x x}$ ) was used for the imaging. The electric field was applied along the $y$ axis, which is parallel to the ferroelectric polarization direction. $\mathbf{b}$, $\mathbf{c}$ SHG images at $10 \mathrm{~K}$ (the bulk AF2 phase) obtained after field cooling at $+240 \mathrm{kV} / \mathrm{m}$ in fields of $\mathbf{b}+240 \mathrm{kV} / \mathrm{m}$ and $\mathbf{c}-240 \mathrm{kV} / \mathrm{m}$. $\mathbf{d}-\mathbf{f}$ Electric field dependence of the SHG images at $10 \mathrm{~K}$ after zero-field cooling obtained in fields of $\mathbf{d} 0 \mathrm{kV} / \mathrm{m}, \mathbf{d}+240 \mathrm{kV} / \mathrm{m}$, and $\mathbf{e}-240 \mathrm{kV} / \mathrm{m}$. Scale bars are $200 \mu \mathrm{m}$. 
surface strain, which may be present in the vicinity of crystallographic defects may suppress the phase transition from the AF2 phase to the AF1 phase, which would then stabilize a spatially confined multiferroic state in its bulk non-multiferroic environment. In the supplementary, we show that in the present sample grooves as a result of the sample polishing are the most likely source of such strain.

In contrast to this accidental occurrence, the coupling between strain and multiferroicity allows us to create the multiferroic phase at specified locations with a scriber. In Fig. 4a, we show an SHG image in the non-multiferroic AF1 phase of a new (001)oriented sample that clearly demonstrates our ability to write multiferroic structures in a highly controlled manner. We propose a model for the locally restricted AF2 phase in Fig. 4b. Interestingly, the phase boundary between the bulk-AF1 phase and the confined AF2 phase does not require a transitional region of finite width for this model magnetic structure. When entering the confined AF2 phase from the collinear AF1 phase, the spins immediately begin to rotate from unit cell to unit cell to reveal the multiferroic AF2 spin spiral. Note that in contrast to the $\mathrm{AF} 1-\mathrm{AF} 2$ boundary, the AF2-AF2 domain walls exhibit width on the order of $10 \mathrm{~nm}^{29}$.

To conclude, we have experimentally proven the existence of multiferroic regions in the form of ribbons within a nonmultiferroic environment for which strain is likely responsible. We confirmed that these ribbons stabilize domains with opposite electric polarization which is robust against external electric
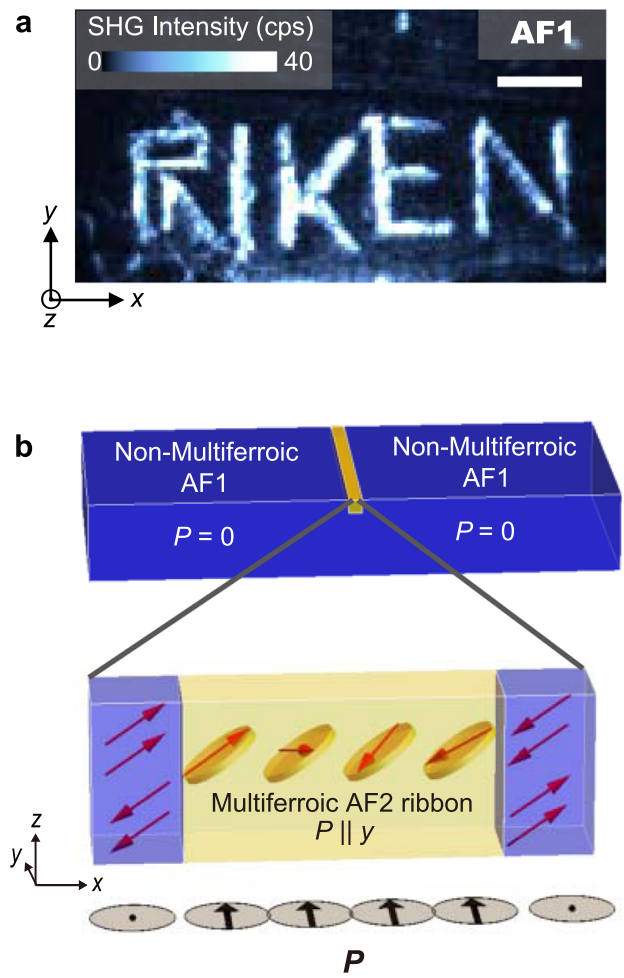

Fig. 4 Controlled writing of the multiferroic state into a non-multiferroic environment. a SHG image at the photon energy $2 \hbar \omega=2.1 \mathrm{eV}$ obtained at $6.5 \mathrm{~K}$ in the non-multiferroic AF1 phase. Letters were drawn onto the crystal surface with a scriber. Scale bar, $200 \mu \mathrm{m}$. b Model of the AF1/AF2 phase coexistence. The bulk magnetic structure is of the collinear non-multiferroic AF1 type, whereas the spatially confined ribbons show the AF2 multiferroic spiral spin structure. Note that the width of the AF1-AF2 phase boundary can be zero as the onset of the magnetic spiral and, hence, of the magnetically induced electric polarization occurs immediately after crossing from the non-multiferroic into the multiferroic phase. fields. The proposed strain-multiferroic coupling enabled us to write spatially controlled multiferroic patterns into a nonmultiferroic template, thus establishing a highly controlled scheme to seed magnetoelectric functionalities at the local level.

\section{Methods}

Sample preparation. Single crystals of $\mathrm{MnWO}_{4}$ were grown by the floating zone melting method. Stoichiometric amounts of powders of $\mathrm{MnO}(5.533 \mathrm{~g})$ and $\mathrm{WO}_{3}$ $(18.083 \mathrm{~g})$ were mixed. A polycrystalline rod of $\mathrm{MnWO}_{4}$ was synthesized by a solid-state reaction by heating at $1000^{\circ} \mathrm{C}$ for 18 hours in the air. The single-crystal growth was carried out in airflow with a feed speed of $3 \mathrm{~mm} / \mathrm{h}$ by using an infrared radiation furnace. The obtained crystal was oriented using Laue X-ray diffraction patterns and cut into thin plates with the widest faces (001). Sample thickness is $200 \mu \mathrm{m}$ for the measurements in Figs. 1-3, whereas it is $1.7 \mathrm{~mm}$ for the SHG image in Fig. 4a. The crystal defects on the sample surface were naturally applied during the polishing procedure for the former sample, whereas we intentionally applied them for the latter sample by using a scriber at room temperature. The scriber was installed on an XYZ stage with a precise position adjustment, which allows for writing the letters at specific positions on the sample surface.

SHG imaging. The light source used for our SHG imaging measurements is a regeneratively amplified laser, producing $190 \mathrm{fs}$ light pulses at $6 \mathrm{kHz}$ repetition rate The wavelength of the laser was tuned with an optical parametric amplifier. The sample was mounted on a copper holder with a hole diameter $1 \mathrm{~mm}$ in a liquidhelium-cooled variable-temperature cryostat. The SHG data points and images were taken in a transmission geometry using a $200 \mathrm{~mm}$ telephoto lens for projection and a liquid-nitrogen-cooled CCD camera as a detector.

Reporting summary. Further information on research design is available in the Nature Research Reporting Summary linked to this article.

\section{Data availability}

The data that support the findings of this study are available from the corresponding authors on reasonable request.

Received: 13 April 2021; Accepted: 29 September 2021; Published online: 27 October 2021

\section{References}

1. Balents, L. Spin liquids in frustrated magnets. Nature 464, 199-208 (2010)

2. Kurumaji, T. et al. Skyrmion lattice with a giant topological Hall effect in a frustrated triangular-lattice magnet. Science 365, 914-918 (2019).

3. Kimura, T. et al. Magnetic control of ferroelectric polarization. Nature 426, 55-58 (2003)

4. Fiebig, M., Lottermoser, T., Meier, D. \& Trassin, M. The evolution of multiferroics. Nat. Rev. Mater. 1, 16046 (2016).

5. Tokura, Y. \& Seki, S. Multiferroics with spiral spin orders. Adv. Mater. 22 1554-1565 (2010)

6. Hwang, H. Y. et al. Emergent phenomena at oxide interfaces. Nat. Mater. 11, 103-113 (2012).

7. Mannhart, J. \& Schlom, D. G. Oxide interfaces-an opportunity for electronics. Science 327, 1607-1611 (2010).

8. Meier, D. Functional domain walls in multiferroics. J. Phys. Condens. Matter 27, 463003 (2015).

9. Meier, D. et al. Anisotropic conductance at improper ferroelectric domain walls. Nat. Mater. 11, 284-288 (2012).

10. Schirhagl, R., Chang, K., Loretz, M. \& Degen, C. L. Nitrogen-vacancy centers in diamond: nanoscale sensors for physics and biology. Annu. Rev. Phys. Chem. 65, 83-105 (2014).

11. Ohtomo, A. \& Hwang, H. Y. A high-mobility electron gas at the LaAlO3/ SrtiO3 heterointerface. Nature 427, 423-426 (2004).

12. Aird, A. \& Salje, E. K. H. Sheet superconductivity in twin walls: experimental evidence of $\mathrm{WO}_{3-\mathrm{x}}$. J. Phys. Condens. Matter 10, L377-L380 (1998).

13. Hassanpour, E. et al. Interconversion of multiferroic domains and domain walls. Nat. Commun. 12, 2755 (2021).

14. Logginov, A. S. et al. Room temperature magnetoelectric control of micromagnetic structure in iron garnet films. Appl. Phys. Lett. 93, 182510 (2008).

15. Lautenschläger, G. et al. Magnetic phase transitions of $\mathrm{MnWO}_{4}$ studied by the use of neutron diffraction. Phys. Rev. B 48, 6087 (1993).

16. Ehrenberg, H. et al. Magnetic phase diagrams of $\mathrm{MnWO}_{4}$. J. Phys. Condens Matter 9, 3189-3203 (1997). 
17. Katsura, H., Nagaosa, N. \& Balatsky, A. V. Spin current and magnetoelectric effect in noncollinear magnets. Phys. Rev. Lett. 95, 1-4 (2005).

18. Sergienko, I. A. \& Dagotto, E. Role of the Dzyaloshinskii-Moriya interaction in multiferroic perovskites. Phys. Rev. B Condens. Matter Mater. Phys. 73, 094434 (2006).

19. Taniguchi, K., Abe, N., Takenobu, T., Iwasa, Y. \& Arima, T. Ferroelectric polarization flop in a frustrated magnet $\mathrm{MnWO}_{4}$ induced by a magnetic field. Phys. Rev. Lett. 97, 097203 (2006).

20. Arkenbout, A. H., Palstra, T. T. M., Siegrist, T. \& Kimura, T. Ferroelectricity in the cycloidal spiral magnetic phase of $\mathrm{MnWO}_{4}$. Phys. Rev. B 74, 184431 (2006).

21. Birss, R. R. Symmetry and Magnetism. (North Holland Publishing Company, 1966).

22. Meier, D. et al. Second harmonic generation on incommensurate structures: the case of multiferroic MnWO4. Phys. Rev. B 82, 155112 (2010).

23. Meier, D. et al. Observation and coupling of domains in a spin-spiral multiferroic. Phys. Rev. Lett. 102, 107202 (2009).

24. Meier, D. et al. Topology and manipulation of multiferroic hybrid domains in $\mathrm{MnWO}_{4}$. Phys. Rev. B 80, 224420 (2009).

25. Shen, Y. R. The Principles of Nonlinear Optics. (John Wiley \& Sons, New York, 1984).

26. Leo, N., Meier, D., Becker, P., Bohatý, L. \& Fiebig, M. Magnetically driven second-harmonic generation with phase matching in $\mathrm{MnWO}_{4}$. Opt. Express 23, 27700 (2015)

27. Boyd, R. W. Nonlinear optics. 3rd edn. (Academic, 2008).

28. Chaudhury, R. P. et al. Thermal expansion and pressure effect in $\mathrm{MnWO}_{4}$. Phys. B Condens. Matter 403, 1428-1430 (2008).

29. Leo, N. et al. Polarization control at spin-driven ferroelectric domain walls. Nat. Commun. 6, 6661 (2015).

\section{Acknowledgements}

S.T. is supported by Grant-in-Aid for Scientific Research from JSPS, Japan (grant no. JP18K14154 and JP20H01867). M.F. thanks the SNSF for financial support under grant no. 200021_178825 and he appreciates sabbatical support by the ETH and the CEMS at RIKEN. N.O. is supported by PRESTO JST (no. JPMJPR17I3). The authors thank Dr. Thomas Weber of ETH Zurich for carrying out high-resolution x-ray diffractometry on the MnWO4 samples with applied defects.

\section{Author contributions}

M.F. and N.O. conceived and guided the project. S.T. set up the SHG imaging measurements with the help of M.F. and N.O. S.T. prepared the samples and carried out the
SHG measurements. L.F. performed the SHG measurements on the samples with applied defects. S.T., M.F., L.F., T.-h.A., Y.T., and N.O. jointly interpreted the data and wrote the manuscript.

\section{Competing interests}

The authors declare no competing interests.

\section{Additional information}

Supplementary information The online version contains supplementary material available at https://doi.org/10.1038/s41467-021-26451-0.

Correspondence and requests for materials should be addressed to Shingo Toyoda or Manfred Fiebig.

Peer review information Nature Communications thanks Liuyan Zhao and the other, anonymous, reviewer(s) for their contribution to the peer review of this work.

Reprints and permission information is available at http://www.nature.com/reprints

Publisher's note Springer Nature remains neutral with regard to jurisdictional claims in published maps and institutional affiliations.

(c) (i) Open Access This article is licensed under a Creative Commons Attribution 4.0 International License, which permits use, sharing, adaptation, distribution and reproduction in any medium or format, as long as you give appropriate credit to the original author(s) and the source, provide a link to the Creative Commons license, and indicate if changes were made. The images or other third party material in this article are included in the article's Creative Commons license, unless indicated otherwise in a credit line to the material. If material is not included in the article's Creative Commons license and your intended use is not permitted by statutory regulation or exceeds the permitted use, you will need to obtain permission directly from the copyright holder. To view a copy of this license, visit http://creativecommons.org/ licenses/by/4.0/.

(C) The Author(s) 2021 